\title{
Bioinformatics analysis identifies several intrinsically disordered human E3 ubiquitin-protein ligases
}

Wouter Boomsma, Sofie V Nielsen, Kresten Lindorff-Larsen, Rasmus Hartmann-Petersen, Lars Ellgaard

The ubiquitin-proteasome system targets misfolded proteins for degradation. Since the accumulation of such proteins is potentially harmful for the cell, their prompt removal is important. E3 ubiquitin-protein ligases mediate substrate ubiquitination by bringing together the substrate with an E2 ubiquitin-conjugating enzyme, which transfers ubiquitin to the substrate. For misfolded proteins, substrate recognition is generally delegated to molecular chaperones that subsequently interact with specific E3 ligases. An important exception is San1, a yeast E3 ligase. San1 harbors extensive regions of intrinsic disorder, which provide both conformational flexibility and sites for direct recognition of misfolded targets of vastly different conformations. So far, no mammalian ortholog of San 1 is known, nor is it clear whether other E3 ligases utilize disordered regions for substrate recognition. Here, we conduct a bioinformatics analysis to examine $>600$ human and $\mathrm{S}$. cerevisiae E3 ligases to identify enzymes that are similar to San1 in terms of function and/or mechanism of substrate recognition. An initial sequence-based database search was found to detect candidates primarily based on the homology of their ordered regions, and did not capture the unique disorder patterns that encode the functional mechanism of San1. However, by searching specifically for key features of the Sanl sequence, such as long regions of intrinsic disorder embedded with short stretches predicted to be suitable for substrate interaction, we identified several E3 ligases with these characteristics. Our initial analysis revealed that another remarkable trait of San1 is shared with several candidate E3 ligases: long stretches of complete lysine suppression, which in San1 limits auto-ubiquitination. We encode these characteristic features into a San1 similarity-score, and present a set of proteins that are plausible candidates as San1 counterparts in humans. In conclusion, our work indicates that San1 is not a unique case, and that several other yeast and human E3 ligases have sequence properties that may allow them to recognize substrates by a similar mechanism as San1. 


\section{Bioinformatics analysis identifies several intrinsically disordered human E3 ubiquitin-protein ligases}

Wouter Boomsma $^{1 *}$, Sofie V. Nielsen ${ }^{1}$, Kresten Lindorff-Larsen ${ }^{1}$, Rasmus Hartmann-Petersen ${ }^{1}$ and Lars Ellgaard ${ }^{1 *}$

${ }^{1}$ Department of Biology, University of Copenhagen, Copenhagen, Denmark

* Corresponding authors

E-mail: lellgaard@bio.ku.dk(LE)

E-mail: wb@bio.ku.dk (WB)

Address:

Department of Biology, University of Copenhagen, Ole Maaløes Vej 5, 2200 Copenhagen N, Denmark 


\section{Abstract}

2 The ubiquitin-proteasome system targets misfolded proteins for degradation. Since the

3 accumulation of such proteins is potentially harmful for the cell, their prompt removal is

4 important. E3 ubiquitin-protein ligases mediate substrate ubiquitination by bringing together the

5 substrate with an E2 ubiquitin-conjugating enzyme, which transfers ubiquitin to the substrate.

6 For misfolded proteins, substrate recognition is generally delegated to molecular chaperones that

7 subsequently interact with specific E3 ligases. An important exception is San1, a yeast E3 ligase.

8 San1 harbors extensive regions of intrinsic disorder, which provide both conformational

9 flexibility and sites for direct recognition of misfolded targets of vastly different conformations.

10 So far, no mammalian ortholog of San1 is known, nor is it clear whether other E3 ligases utilize

11 disordered regions for substrate recognition. Here, we conduct a bioinformatics analysis to

12 examine $>600$ human and $S$. cerevisiae E3 ligases to identify enzymes that are similar to San 1 in

13 terms of function and/or mechanism of substrate recognition. An initial sequence-based database

14 search was found to detect candidates primarily based on the homology of their ordered regions,

15 and did not capture the unique disorder patterns that encode the functional mechanism of San1.

16 However, by searching specifically for key features of the San1 sequence, such as long regions

17 of intrinsic disorder embedded with short stretches predicted to be suitable for substrate

18 interaction, we identified several E3 ligases with these characteristics. Our initial analysis

19 revealed that another remarkable trait of San1 is shared with several candidate E3 ligases: long

20 stretches of complete lysine suppression, which in San1 limits auto-ubiquitination. We encode

21 these characteristic features into a San1 similarity-score, and present a set of proteins that are

22 plausible candidates as San1 counterparts in humans. In conclusion, our work indicates that San1 
23 is not a unique case, and that several other yeast and human E3 ligases have sequence properties

24 that may allow them to recognize substrates by a similar mechanism as San1.

\section{Introduction}

26 A coordinated and efficient regulation of protein levels is of crucial importance to all cells, and

27 ubiquitin-mediated proteasomal degradation plays a central role in protein homeostasis. In

28 addition to its role in controlling protein levels for regulatory purposes, the proteasome also

29 degrades misfolded proteins that may otherwise accumulate as toxic protein aggregates.

30 Cellular proteins are targeted for proteasomal degradation by the attachment of multiple

31 moieties of the small regulatory protein ubiquitin, most often to lysine residues, but in some

32 cases also to serines, threonines or cysteines (Wang et al. 2012). This process occurs through the

33 sequential action of a ubiquitin-activating enzyme (a so-called E1 enzyme), a ubiquitin-

34 conjugating enzyme (E2) and a ubiquitin-protein ligase (E3). First, an E1 enzyme gets activated

35 with a ubiquitin moiety in an ATP-dependent process, and subsequently passes on ubiquitin to an

36 E2 enzyme. The E2 enzyme then interacts with an E3 ubiquitin-protein ligase. The most

37 common class of E3 ligases contains a Really Interesting New Gene (RING) domain, which acts

38 to bind ubiquitin-conjugating E2 enzymes (Metzger et al. 2014). RING E3 ligases thus bridge

39 ubiquitin-charged E2s with substrates, which then become ubiquitinated. Multiple rounds of this

40 process ensure substrate poly-ubiquitination, a requirement for degradation of most proteasomal

41 substrates (for a recent review of the ubiquitin-proteasome system see (Kleiger \& Mayor 2014)).

$42 \quad$ Although most components and the basic overall mechanisms of this system are well

43 understood, we still lack a comprehensive molecular understanding of how proteins are

44 recognized and marked for degradation. In some cases, E3 enzymes bind ancillary protein factors 
45 that aid in the recognition of the substrate. The E3 enzyme CHIP, for example, ubiquitinates

46 misfolded proteins that are recognized and delivered to CHIP via Hsp70 chaperones (Arndt et al.

47 2007). In this case, recognition of partially folded or misfolded substrates is offloaded to a

48 chaperone, and the E3 enzyme then recognizes the substrate-loaded Hsp70.

49 The use of protein co-factors to aid in substrate recognition is one solution to the problem

50 of how a fixed number of E3 enzymes can recognize the very large and conformationally diverse

51 set of substrates that the system must be able to deal with. Recently, however, an alternative

52 mechanism has been suggested for how the San1 E3 ligase from Saccharomyces cerevisiae can

53 recognize misfolded substrates. San1, a protein of 610 amino acids with a RING domain

54 spanning residues 165-280, appears to be largely devoid of well-defined three-dimensional

55 structure (Rosenbaum et al. 2011). Although the cytosolic Hsp70 chaperone Ssal and the ring-

56 shaped Cdc48/p97 chaperone can assist in degradation of San1 substrates (Gallagher et al. 2014;

57 Guerriero et al. 2013; Kriegenburg et al. 2014), a number of biochemical experiments strongly

58 suggest that the disordered parts of the San1 sequence provide direct binding sites for substrate

59 proteins targeted for degradation (Rosenbaum et al. 2011). In particular, throughout the

60 disordered regions of San1 there are several short hydrophobic stretches that function as

61 substrate binding sites (Rosenbaum et al. 2011). This distribution of substrate binding patches,

62 combined with the high flexibility of the disordered protein, allows San 1 to interact with

63 multiple different misfolded proteins and bypasses the need for chaperones. Hence, the intrinsic

64 disorder present in San 1 apparently underlies its ability to recognize misfolded proteins.

65 However, potentially due to the poor sequence conservation of disordered proteins, it has not yet

66 been possible to identify any San1 orthologs in higher eukaryotes. 
Intrinsic disorder in proteins is now recognized to be broadly distributed across all

68

kingdoms of life, and to play an important role in a large number of biological processes (Dyson $\&$ Wright 2005). The large flexibility of fully intrinsically disordered proteins, or long stretches of intrinsic disorder in conjunction with well-folded domains, is believed to provide a number of particular properties to proteins (Babu et al. 2012). Importantly, the heterogeneous structures attained by these proteins allow them to bind a broad range of target proteins, either through a process called folding-upon-binding in which the disordered regions gain a specific structure upon binding to a target, or through the formation of "fuzzy complexes" in which substantial disorder is retained (Cumberworth et al. 2013).

The finding that San 1 can recognize its protein substrates directly via its intrinsically disordered regions prompted us to explore whether this mechanism might be employed by other E3 enzymes. Using sequence conservation as a search criterion proved to be insufficient in this respect, presumably due to the low levels of sequence conservation that characterize intrinsically disordered protein regions. As an alternative, site-specific disorder profiles have been shown to display substantial preservation through evolution (Brown et al. 2010; Brown et al. 2011; Chen et al. 2006a; Chen et al. 2006b; Daughdrill et al. 2007), and have recently been used as the basis for detecting similar disorder functionalities across species (Mahani et al. 2013; Petrovich et al. 2015). We employ such an approach here, by constructing a procedure that searches for disorder properties similar to those of San1, combined with information about the unique pattern of binding regions known from the San1 protein. A previous study of structural disorder in the human ubiquitin-proteasome system found evidence for disorder in E1, E2 and - to the greatest extent - E3 enzymes, and provided examples for how disordered regions in E3 enzymes can function either as flexible linker regions or sometimes as sites for interaction with folded 
90 domains in substrates (Bhowmick et al. 2013). Our work extends this analysis by focusing on the

91 extent to which E3 enzymes may use their intrinsic disorder in direct substrate recognition. The

92 method was applied on datasets of 563 human and 80 yeast E3 enzymes. We demonstrate that a

93 number of human E3 enzymes, in addition to displaying pervasive protein disorder, show two

94 other hallmarks of the San1 sequence: short stretches of higher order potentially involved in

95 substrate binding and a remarkably low content of lysine residues. Overall, our data indicate that

96 San1 is not a unique case among E3 ligases and that a number of these enzymes could display a

97 substrate recognition mode similar to San1.

98 Results

99 Sequence-conservation based analyses reveal no obvious human

100 orthologs of San1

101 As of yet, no human ortholog of yeast San1 is known. In a first attempt to identify such a protein,

102 we focused on the unusual RING domain of San1. Whereas a classical RING domain has a

103 consensus motif of Cys- $\mathrm{X}_{2}-\mathrm{Cys}-\mathrm{X}_{9-39}-\mathrm{Cys}-\mathrm{X}_{1-3}-\mathrm{His}-\mathrm{X}_{2-3}-\mathrm{Cys} / \mathrm{Asn} / \mathrm{His}-\mathrm{X}_{2}-\mathrm{Cys}-\mathrm{X}_{4-48}-\mathrm{Cys}_{2}-\mathrm{X}_{2}-\mathrm{Cys}$

104 (where X represents any amino acid residue) (Budhidarmo et al. 2012), the distance between the

105 first Cys- $\mathrm{X}_{2}$-Cys sequence and the following cysteine in the San1 RING domain is 88 residues

106 (in S. cerevisiae), making it unusually long. However, using MOTIF search

107 (http://www.genome.jp/tools/motif/), an algorithm designed to detect a specific sequence motif,

108 we did not find obvious San1 candidates among the human proteins identified. Moreover, using

109 the S. cerevisiae San1 RING domain in PSI-BLAST and DELTA-BLAST (Altschul et al. 1997)

110 searches we did also not detect any human E3 ligase comprising the same characteristic RING 
111 domain as San1. To increase the sensitivity of the sequence-based search, we proceeded with an

112 HMMSearch protocol, where the San1 sequence was encoded into a hidden Markov model using

113 the jackhmmer program (both part of the HMMer software package; http://hmmer.org/). This

114 procedure detected a number of potential matches, but the hits were predominantly defined by

115 similarities in the ordered regions of San1 - primarily the RING domain, and the detected

116 homologues did not generally display the disorder and binding patterns characteristic of San1

117 (Figure S1). In light of these results, we decided to search for human E3 ligases that contain

118 characteristic sequence features of San1 other than those associated with the RING domain.

\section{Initial San1-similarity analysis}

120 Although San1 from S. cerevisiae is largely intrinsically disordered outside of the RING domain,

121 the flexible parts are predicted to contain short interspersed regions of higher order that coincide

122 with sequence conservation among orthologs in other yeast species (Rosenbaum et al. 2011).

123 Experimentally, it has been verified that these more ordered regions confer substrate binding

124 (Rosenbaum et al. 2011). The overall model that emerges is therefore one where multiple

125 binding sites (independently or in conjunction) can engage the substrates, whereas the flexibility

126 provided by the intrinsic disorder allows San1 to adopt its structure to a wide variety of

127 misfolded substrates (Rosenbaum \& Gardner 2011). We therefore analyzed datasets of $S$.

128 cerevisiae and human E3s to identify E3 enzymes containing the same overall disorder/order

129 signature as San1, in addition to a high overall content of intrinsic disorder. Specifically, we

130 employed the consensus disorder annotation from the MobiDB database (Potenza et al. 2015) to

131 identify regions of disorder in E3 enzymes (Figure 1a, Table S1). For the San1 sequence, 78\%

132 was predicted to be disordered (Figure 1b, Table S1). 
We used the ANCHOR predictor (Meszaros et al. 2009), which has also been used in the

134 characterization of San1 (Rosenbaum et al. 2011), to identify short regions within the disordered

135 parts of the E3 enzymes with a potential for molecular interactions. Using only the amino acid

136 sequence, ANCHOR predicts potential interaction sites present within disordered regions. When

137 employing a 0.5 threshold level, $53 \%$ of all residues in San1 were predicted to be ANCHOR

138 positive, with only four of the human E3 proteins scoring higher (Table S1). In the case of San1,

139 the regions of higher order that coincide with sequence conservation among orthologs are

140 generally 15-40 residues long (Rosenbaum et al. 2011). To create an output to better detect a

141 disorder/order pattern similar to the one in San1, we therefore identified the total number of

142 residues contained in ANCHOR positive regions of length 15-40 in each protein of the datasets,

143 and normalized it to the length of the protein.

144 Plotting this ANCHOR score against the consensus disorder, and considering the

145 candidates with highest scores in both dimensions, we indeed found San 1 at the top of the yeast

146 dataset, accompanied by a few other high-scoring candidates (e.g. Slx8; Figure 2, Figure 3). The

147 disorder and ANCHOR profiles for several of the top candidates in the human dataset (e.g. Rnf6,

148 Rnf12, Pja1, Rn111; Figure 2, Figure 3) showed clear similarities to the San1 profile, with

149 general high levels of disorder and the distinct ANCHOR propensity pattern. This initial

150 approach, based on a simple combination of disorder and anchor score, however, had a few

151 shortcomings. First of all, it relies on globally high values of disorder, thereby excluding proteins

152 containing one or more ordered domains. Secondly, it fails to take into account another

153 remarkable feature of San1: long segments without a single occurrence of lysine residues. Both

154 these limitations are addressed below. 


\section{San1-like E3s show substantial lysine suppression}

156 San1 has previously been reported to contain unusually few lysine residues (Fredrickson et al.

157 2013) (Figure 3 and Table S1). Since lysine is the most commonly used residue for ubiquitin

158 conjugation, and based on experimental evidence that the introduction of lysines significantly

159 destabilized the protein in cells, it was proposed that the low level of lysines in San 1 protects the

160 protein from auto-ubiquitination and therefore from degradation (Fredrickson et al. 2013). The

161 few lysines present in San1 are found either within the RING domain or in a short sequence

162 stretch of relatively low disorder immediately adjacent to this domain (Figure 3, black dots), a

163 feature which seems to be shared with several of the highest scoring candidates (Figure 3), in

164 some cases to a remarkable extent (e.g. $>600$ residues were devoid of lysines in the case of

165 RN111). The most significant cases are highlighted in Figure 4, demonstrating that the lysine

166 suppression is strongly conserved among orthologs in a range of different species. The lack of

167 lysines is particularly remarkable in light of the finding that disordered proteins are typically

168 enriched in lysines compared to folded proteins (Tompa 2002). Taken together, these results

169 suggest that the San1-like E3 ligases identified here could employ the same strategy as San1

170 itself to avoid auto-ubiquitination and degradation (but see also Discussion).

\section{Definition of a San1 similarity score}

172 Based on the considerations above, we developed a single score that quantifies whether a protein

173 displays general sequence properties that are similar to those of San1. The score is based on

174 disorder propensity, ANCHOR score and lysine suppression using a sliding window approach.

175 For each protein, every residue is assigned a score based on the three quantities, calculated over a

176 window centered at the residue in question (see Figure 5): a) the percentage of residues with 
177 consensus disorder larger than $0.5, \mathrm{~b}$ ) the percentage of residues within ANCHOR positive

178 stretches, and c) the longest lysine-free stretch of residues, normalized by the length of the

179 window (chosen here as 150 residues). For each position in the sequence, a product of these three

180 quantities is calculated, and each protein is represented with the maximum value ("'the San1

181 similarity score") observed among its residues. Thus, proteins with a high San1 similarity score

182 contain a particular region that simultaneously has a high level of disorder, many potential sites

183 for molecular interaction and strong lysine suppression.

184 The top-scoring candidates based on this approach are presented in Figure 5 (the full list 185 is available as Table S1). As expected, we find that San1 has one of the highest scores, with only

186 a few human candidates scoring higher. In general, we recover many of the candidates found in

187 the initial San1-similarity analysis presented above. As opposed to the initial analysis, the

188 window-based approach allows the identification of larger proteins with a relatively low overall

189 disorder, often reflecting the presence of one or more ordered regions (domains). Two such

190 examples include human Syvn1/Hrd1 and yeast Dma2 (Figure 5). For a further discussion of the

191 choice of parameters and how they affect the San1-similarity score we refer to the Methods

192 section.

\section{Analysis of potential functional orthologs of San1}

194 Based on the bioinformatics investigation described above, we next analyzed specific proteins

195 with high San1 similarity in more detail founded on the current literature. Specifically, among

196 the top scoring hits in Figure 5, we consider those candidates with known links to protein quality

197 control, i.e. the human E3s Syvn1/Hrd1 and Hecw1 and yeast the E3s Doa10, Slx8, and Ufd4.

198 A well-characterized protein appearing in our analysis is human Syvn1/Hrd1, a

199 multispanning transmembrane protein of the ER. The low overall disorder displayed by this 
200 protein is due to the transmembrane region ending at around residue 240 , followed by a

201 disordered C-terminal cytosolic region, which is also low in lysine content (Figure 5). Curiously,

202 the cytosolic tail of the budding yeast protein contains more lysines than in its orthologs.

203 Whether this difference reflects a basic difference in the respective functional mechanisms is 204 presently unknown.

205 Syvn1/Hrd1 is of particular interest in the current context, because it is known to interact 206 with misfolded proteins. Thus, Syvn1/Hrd1 is involved in targeting misfolded ER and secretory

207 proteins for proteasomal degradation in the cytosol through a process known as ER-associated 208 degradation (ERAD) (Christianson \& Ye 2014). In this process, misfolded proteins are first 209 recognized by ER lumenal chaperones and retrotranslocated across the ER membrane. When 210 emerging in the cytosol, substrate proteins are ubiquitinated by ER-membrane localized E3

211 ligases, such as Syvn1/Hrd1 before they are finally degraded by the $26 \mathrm{~S}$ proteasome

212 (Christianson \& Ye 2014). Along with the RING domain, the predicted intrinsically disordered

213 area in HRD1 faces the cytosol, and has so far not been ascribed any particular function.

214 Presumably, Syvn1/Hrd1 transiently engages the at least partially extended ERAD substrates

215 during ubiquitination, and the disordered region could therefore play a role, similar to the 216 situation for San1, in this function.

217 Intriguingly, another ERAD E3, Doa10, was one of the best scoring yeast E3s. Like 218 Syvn1/Hrd1, Doa10 is a multispanning transmembrane protein localized to the ER and inner 219 nuclear membrane where targets a variety of misfolded proteins, e.g. squalene monooxygenase, 220 and enzyme involved in sterol homeostasis (Foresti et al. 2013), for proteasomal degradation 221 ((Habeck et al. 2015) and references therein). As with Syvn1/Hrd1 the disordered region of 
222 Doa10 faces the cytosol, which fits well with its established role in targeting also soluble nuclear

223 and cytosolic proteins for degradation (Ravid et al. 2006).

224 Slx8 is a well-characterized SUMO-targeting ubiquitin ligase (STUbL). In the yeast

225 nucleus, Slx8 forms a heterodimer with Slx5 (Yang et al. 2006) and targets a number of

226 sumoylated substrates for ubiquitin-dependent degradation (Uzunova et al. 2007). At least part of

227 the disordered region of Slx8 is required for heterodimer formation with Slx 5 (Westerbeck et al.

228 2014; Yang et al. 2006), which contains the SUMO interacting motifs that are required for

229 substrate recognition. Similar to San1, the Slx5-Slx8 heterodimer has been shown to participate

230 in protein quality control. Both $S L X 5$ and $S L X 8$ were identified as suppressors of a temperature

231 sensitive mutant in the DNA binding protein Motl (Wang et al. 2006). At high temperatures, the

232 mutant Mot1 protein is highly unstable, but stabilized in $S L X 5$ and $S L X 8$ null mutants (Wang et

233 al. 2006). This is similar to how San 1 was first connected to protein quality control of the mutant

234 proteins Sir4-9 and Cdc68-1 (Gardner et al. 2005), and suggests that the mutant Mot1 protein is

235 still functional at the restrictive temperature, but targeted for degradation. However, the

236 degradation of Mot1 was also shown to depend on the SUMO-ligases Siz1 and Siz2 (Wang et al.

237 2006), revealing that sumoylation of Mot1 was required for Slx5-Slx8 dependent degradation.

238 Although in sequence, they do not appear highly conserved, the mammalian STUbL Rnf4/Snurf

239 is a functional ortholog of budding yeast Slx5-Slx8 (Prudden et al. 2007; Sriramachandran \&

240 Dohmen 2014). However, as opposed to the situation with Slx5-Slx8 in yeast, Rnf4/Snurf forms

241 a homodimer (Plechanovova et al. 2011), which has primarily been connected with the SUMO-

242 dependent degradation of the Pml-Rara oncoprotein (Tatham et al. 2008). Of note, Rnf4/Snurf

243 did not display a high San1-similarity score. However, another less characterized human STUbL,

244 Rn111 (Figure 5), which is also involved in targeting Pml-Rara for SUMO-dependent 
245 degradation (Erker et al. 2013), did show similarity to San1. In contrast to Rnf4/Snurf, Rn111

246 has also been shown to promote non-proteolytic, K63-linked ubiquitination of SUMO-modified

247 substrate proteins (Poulsen et al. 2013).

248 In addition to Syvn1/Hrd1, Doa10 and Slx8 described above, also Hecw1 and Ufd4 have

249 been connected with protein quality control. Specifically, Hecw1, also known as NEDL1, was

250 found to ubiquitinate amyotrophic lateral sclerosis (ALS)-linked SOD1 variants, but not wild

251 type SOD1 (Miyazaki et al. 2004). However, it is not clear that Hecw1 catalyzed SOD1

252 ubiquitination leads to proteasomal degradation. Genetic experiments have also linked the yeast

253 E3 Ufd4 to protein quality control (Theodoraki et al. 2012), and showed that Ufd4 as a

254 proteasome-associated E3 (Xie \& Varshavsky 2002), targets an orphan ER-membrane protein for 255 degradation (Ravid \& Hochstrasser 2007).

\section{Discussion}

257 The recognition and degradation of misfolded proteins by the ubiquitin-proteasome system is of

258 fundamental cellular importance. Only recently has it become clear that substrate recognition

259 does not always rely on molecular chaperones, but can also occur through a direct binding

260 mechanism involving intrinsic disorder, as employed by San1. We reasoned that this mechanism

261 might well be employed by other E3 ligases, featuring sequence characteristics similar to San1

262 itself. While San1 is exclusively located in the nucleus (Gardner et al. 2005), the substrate

263 recognition mechanism employed by this protein could in principle also be employed by E3

264 ligases of the cytosol. Still, most of the proteins we identify as being San1-like have been shown

265 to localize to the nucleus (Her \& Chung 2009; Liu et al. 2006; Xu et al. 2009). The underlying

266 reason for this apparent bias is presently unknown, although nuclear proteins in general appear to

267 be enriched for disorder as compared to, for example, cytosolic proteins (Ward et al. 2004), and 
268 in addition the nucleus appears to be a particularly active organelle in regard to protein quality

269 control (Nielsen et al. 2014; Park et al. 2013).

270 In the case of San1, it has been shown that one reason for its unusually low content of

271 lysine residues is to protect it from auto-ubiquitination and untimely proteasomal degradation

272 (Fredrickson et al. 2013). Many of the disordered San1-like E3s that we identify here also

273 display long stretches devoid of lysine residues. For instance, Rnf6 and Rnf12 both contain a

274 remarkably long stretch of around 500 residues devoid of lysines, a feature conserved among

275 their orthologs (Figure 4). It is therefore possible that these proteins employ the same strategy as

276 San1 to avoid auto-ubiquitination and degradation. Indeed, recent work shows a clear propensity

277 for ubiquitinated lysines in proteasomal substrates to occur in regions of intrinsic disorder, and

278 moreover these lysines are often located in close proximity to segments of $>19$ consecutive

279 disordered residues (Guharoy et al. 2016). However, since lysine residues can undergo numerous

280 post-translational modifications, including for instance sumoylation and acetylation, the low

281 lysine content need not necessarily be connected with ubiquitination and degradation. Indeed, for

282 Dma2, auto-ubiquitination followed by degradation was shown to occur despite the presence of a

283 region with low lysine content (Loring et al. 2008).

284 It is noteworthy that both Rn111 and Slx8 belong to the rather small family of STUbL-

285 type E3s. Since data suggest that several proteins are conjugated to SUMO in response to heat

286 shock (Tammsalu et al. 2014; Tatham et al. 2011), such STUbL E3s are likely to be important

287 for protein quality control. Indeed, like San1, Slx 8 has been linked to protein quality control

288 (Wang et al. 2006). However, since the SUMO interacting motifs that are found in STUbLs are

289 generally located in intrinsically disordered regions (Vogt \& Hofmann 2012), it cannot be ruled

290 out that our approach is biased to detect STUbLs. 
The concept that disorder can function to target protein misfolding in protein quality

292 control is not only known from the case of San1. Recently, a disordered region of the cytosolic

293 Bag6 protein, which functions to maintain misfolded aggregation-prone ERAD substrates in

294 solution en route to the proteasome (Wang et al. 2011), has been demonstrated to bind and

295 prevent aggregation of unfolded luciferase in vitro (Xu et al. 2013). Moreover, it has recently

296 become clear that Bag6 harbors multiple "hydrophobicity recognizing" modules for binding of

297 various substrates (Tanaka et al. 2015). Notably, when applying our procedure to the human

298 Bag6 sequence we find a San1-similarity score of 0.51 (Figure S2), similar to the highest scoring

299 E3s in Figure 5 (see Table S1). This suggests that our approach could be useful also for

300 identifying other components of the cellular degradation machinery. In the case of Bag6, we

301 speculate that the low lysine content present in the protein, although it is not itself an E3 ligase,

302 protects the protein from ubiquitination by E3 ligases, such as RNF126, with which Bag6

303 directly interacts (Rodrigo-Brenni et al. 2014). Another recent example shows that a disordered

304 C-terminal portion of the Ube2w E2 enzyme recognizes only intrinsically disordered N-termini

305 of proteins to catalyze their N-terminal ubiquitination (Vittal et al. 2015). Indeed, we speculate

306 that the San1-like proteins identified here might fall into two different classes. One class, which

307 includes San1, Slx8 and Syvn1/Hrd1, appears to utilize its intrinsic disorder to identify a broader

308 range of misfolded substrates, whereas the other class might rather use disordered regions to help

309 recognize other substrates that perhaps themselves contain disordered regions. The identification

310 here of a number of San1-like human E3 ligases suggests that further examples could be added in

311 the near future, opening up for more detailed functional and biophysical studies of the

312 mechanisms by which intrinsic disorder in E3 enzymes is used to regulate protein homeostasis. 


\section{Methods}

\section{HMM sequence-based search procedure}

315 The hidden Markov model based sequence searches were conducted using the HMMER software

316 package (http://hmmer.org/) by constructing a model using the jackhmmer tool, and

317 subsequently using the hmmsearch program to search through the entire Uniprot databases of

318 Saccharomyces cerevisiae and human, respectively. Default values were used for these

319 programs. The top-10 matches for human and top-5 hits for yeast are included as supplementary

320 information (Figure S1).

321 Datasets

322 For the remaining analyses, we searched directly within a set of 80 yeast E3 ligases (Li et al.

323 2008) and a set of 563 human E3 ligases recently published by Tompa and coworkers

324 (Bhowmick et al. 2013). This set was constructed as a merge of an extract from the KEGG

325 BRITE database (Kanehisa \& Goto 2000) and well-characterized E3 ligases from a previously

326 published dataset (Li et al. 2008), filtered for uncertain annotations, identical gene names, and

327 internal sequence homology $(>85 \%)$, leading to an unbiased, representative set of the currently

328 known E3 proteins. It should be noted, however, that additional human E3 ligases probably exist

329 (Bhowmick et al. 2013; Li et al. 2008), and more than 600 RING-type E3 ligases are likely

330 expressed in human cells (Deshaies \& Joazeiro 2009).

331 All results obtained in the analysis for this paper are made available as an annotation of

332 this original data set, presented as an Excel file in the Supplementary data (Table S1). This

333 dataset contains all position-specific prediction results, it provides the resulting San1 similarity

334 score, and allows for easy sorting of candidates based on the criteria outlined in the paper. The 
335 spreadsheet also permits reanalyses of the data with different parameter values (e.g. the lengths

336 of the sliding window and the ANCHOR stretch).

\section{Prediction of protein binding regions}

338 We used the ANCHOR (Meszaros et al. 2009) program to assign position-specific scores for

339 protein binding within disordered regions. For the purpose of the San1 similarity score, we

340 considered stretches of between 15 and 40 residues (see below) for which the ANCHOR scores

341 are all higher than 0.5 . The total number of residues occurring within such regions was

342 normalized by the length of the window to give an ANCHOR stretch score. No requirements

343 were imposed on the disorder status of such regions.

\section{Choice of parameters}

345 The size of the sliding window and the boundaries defining an ANCHOR stretch (15-40), are

346 free parameters that we chose based on the observed features of San1. The window size for

347 lysine content was set to 150, which is compatible with the particular stretch found in San1.

348 Choosing larger values penalizes smaller proteins (candidates that are smaller than the window

349 size will be assigned a score of zero), and choosing smaller values makes the score less sensitive

350 to long lysine-free stretches. The 15 and 40 residue ANCHOR stretch boundaries were based

351 directly on the lengths observed in San1 (Rosenbaum et al. 2011). We probed the sensitivity of

352 our approach to these choices by considering the top candidates with varying values for both of

353 these parameters and found the presented results to be quite robust, as long as the chosen values

354 were not directly at odds with the characteristics observed for San1. For instance, when changing

355 the ANCHOR stretch length to 5-35, we find that 17 out of 20 of the Top 20 candidates in Table

356 S1 are preserved. 
359

360

361

362

363

364

365

366

367

368

369

370

371

372

373

374

375

376

377

378

379

380

381

382

383

384

385

386

387

388

389

390

391

392

393

394

395

396

397

398

399

400

Altschul SF, Madden TL, Schaffer AA, Zhang J, Zhang Z, Miller W, and Lipman DJ. 1997. Gapped BLAST and PSI-BLAST: a new generation of protein database search programs. Nucleic Acids Res 25:3389-3402.

Arndt V, Rogon C, and Hohfeld J. 2007. To be, or not to be--molecular chaperones in protein degradation. Cell Mol Life Sci 64:2525-2541. 10.1007/s00018-007-7188-6

Babu MM, Kriwacki RW, and Pappu RV. 2012. Structural biology. Versatility from protein disorder. Science 337:1460-1461. 10.1126/science.1228775

Bhowmick P, Pancsa R, Guharoy M, and Tompa P. 2013. Functional diversity and structural disorder in the human ubiquitination pathway. PLoS One 8:e65443. 10.1371/journal.pone.0065443

Brown CJ, Johnson AK, and Daughdrill GW. 2010. Comparing models of evolution for ordered and disordered proteins. Mol Biol Evol 27:609-621. msp277 [pii]

10.1093/molbev/msp277

Brown CJ, Johnson AK, Dunker AK, and Daughdrill GW. 2011. Evolution and disorder. Curr Opin Struct Biol 21:441-446. 10.1016/j.sbi.2011.02.005

Budhidarmo R, Nakatani Y, and Day CL. 2012. RINGs hold the key to ubiquitin transfer. Trends Biochem Sci 37:58-65. 10.1016/j.tibs.2011.11.001

Chen JW, Romero P, Uversky VN, and Dunker AK. 2006a. Conservation of intrinsic disorder in protein domains and families: I. A database of conserved predicted disordered regions. $J$ Proteome Res 5:879-887. 10.1021/pr060048x

Chen JW, Romero P, Uversky VN, and Dunker AK. 2006b. Conservation of intrinsic disorder in protein domains and families: II. functions of conserved disorder. $J$ Proteome Res 5:888898. 10.1021/pr060049p

Christianson JC, and Ye Y. 2014. Cleaning up in the endoplasmic reticulum: ubiquitin in charge. Nat Struct Mol Biol 21:325-335. 10.1038/nsmb.2793

Cumberworth A, Lamour G, Babu MM, and Gsponer J. 2013. Promiscuity as a functional trait: intrinsically disordered regions as central players of interactomes. Biochem $J$ 454:361369. 10.1042/BJ20130545

Daughdrill GW, Narayanaswami P, Gilmore SH, Belczyk A, and Brown CJ. 2007. Dynamic behavior of an intrinsically unstructured linker domain is conserved in the face of negligible amino acid sequence conservation. $J$ Mol Evol 65:277-288. 10.1007/s00239007-9011-2

Deshaies RJ, and Joazeiro CA. 2009. RING domain E3 ubiquitin ligases. Annu Rev Biochem 78:399-434. 10.1146/annurev.biochem.78.101807.093809

Dyson HJ, and Wright PE. 2005. Intrinsically unstructured proteins and their functions. Nat Rev Mol Cell Biol 6:197-208. 10.1038/nrm1589

Erker Y, Neyret-Kahn H, Seeler JS, Dejean A, Atfi A, and Levy L. 2013. Arkadia, a novel SUMO-targeted ubiquitin ligase involved in PML degradation. Mol Cell Biol 33:21632177. 10.1128/MCB.01019-12

Foresti O, Ruggiano A, Hannibal-Bach HK, Ejsing CS, and Carvalho P. 2013. Sterol homeostasis requires regulated degradation of squalene monooxygenase by the ubiquitin ligase Doa10/Teb4. Elife 2:e00953. 10.7554/eLife.00953 
401

402

403

404

405

406

407

408

409

410

411

412

413

414

415

416

417

418

419

420

421

422

423

424

425

426

427

428

429

430

431

432

433

434

435

436

437

438

439

440

441

442

443

444

445

446

Fraschini R, Bilotta D, Lucchini G, and Piatti S. 2004. Functional characterization of Dma1 and Dma2, the budding yeast homologues of Schizosaccharomyces pombe Dma1 and human Chfr. Mol Biol Cell 15:3796-3810. 10.1091/mbc.E04-02-0094

Fredrickson EK, Clowes Candadai SV, Tam CH, and Gardner RG. 2013. Means of selfpreservation: how an intrinsically disordered ubiquitin-protein ligase averts selfdestruction. Mol Biol Cell 24:1041-1052. 10.1091/mbc.E12-11-0811

Gallagher PS, Clowes Candadai SV, and Gardner RG. 2014. The requirement for Cdc48/p97 in nuclear protein quality control degradation depends on the substrate and correlates with substrate insolubility. J Cell Sci 127:1980-1991. 10.1242/jcs.141838

Gardner RG, Nelson ZW, and Gottschling DE. 2005. Degradation-mediated protein quality control in the nucleus. Cell 120:803-815. 10.1016/j.cell.2005.01.016

Gontan C, Achame EM, Demmers J, Barakat TS, Rentmeester E, van IW, Grootegoed JA, and Gribnau J. 2012. RNF12 initiates X-chromosome inactivation by targeting REX1 for degradation. Nature 485:386-390. 10.1038/nature11070

Guerriero CJ, Weiberth KF, and Brodsky JL. 2013. Hsp70 targets a cytoplasmic quality control substrate to the San1p ubiquitin ligase. $J$ Biol Chem 288:18506-18520. 10.1074/jbc.M113.475905

Guharoy M, Bhowmick P, Sallam M, and Tompa P. 2016. Tripartite degrons confer diversity and specificity on regulated protein degradation in the ubiquitin-proteasome system. Nat Commun 7:10239. 10.1038/ncomms10239

Habeck G, Ebner FA, Shimada-Kreft H, and Kreft SG. 2015. The yeast ERAD-C ubiquitin ligase Doa10 recognizes an intramembrane degron. $J$ Cell Biol 209:261-273. $10.1083 /$ jcb. 201408088

Her YR, and Chung IK. 2009. Ubiquitin Ligase RLIM Modulates Telomere Length Homeostasis through a Proteolysis of TRF1. J Biol Chem 284:8557-8566. 10.1074/jbc.M806702200

Iakoucheva LM, Radivojac P, Brown CJ, O'Connor TR, Sikes JG, Obradovic Z, and Dunker AK. 2004. The importance of intrinsic disorder for protein phosphorylation. Nucleic Acids Res 32:1037-1049. 10.1093/nar/gkh253

Kanehisa M, and Goto S. 2000. KEGG: kyoto encyclopedia of genes and genomes. Nucleic Acids Res 28:27-30.

Kleiger G, and Mayor T. 2014. Perilous journey: a tour of the ubiquitin-proteasome system. Trends Cell Biol. 10.1016/j.tcb.2013.12.003

Kriegenburg F, Jakopec V, Poulsen EG, Nielsen SV, Roguev A, Krogan N, Gordon C, Fleig U, and Hartmann-Petersen R. 2014. A chaperone-assisted degradation pathway targets kinetochore proteins to ensure genome stability. PLoS Genet 10:e1004140. 10.1371/journal.pgen.1004140

Li W, Bengtson MH, Ulbrich A, Matsuda A, Reddy VA, Orth A, Chanda SK, Batalov S, and Joazeiro CA. 2008. Genome-wide and functional annotation of human E3 ubiquitin ligases identifies MULAN, a mitochondrial E3 that regulates the organelle's dynamics and signaling. PLoS One 3:e1487. 10.1371/journal.pone.0001487

Liang J, Suhandynata RT, and Zhou H. 2015. Phosphorylation of Sae2 Mediates Forkheadassociated (FHA) Domain-specific Interaction and Regulates Its DNA Repair Function. $J$ Biol Chem 290:10751-10763. 10.1074/jbc.M114.625293

Liu W, Rui H, Wang J, Lin S, He Y, Chen M, Li Q, Ye Z, Zhang S, Chan SC, Chen YG, Han J, and Lin SC. 2006. Axin is a scaffold protein in TGF-beta signaling that promotes degradation of Smad7 by Arkadia. EMBO J 25:1646-1658. 10.1038/sj.emboj.7601057 
447

448

449

450

451

452

453

454

455

456

457

458

459

460

461

462

463

464

465

466

467

468

469

470

471

472

473

474

475

476

477

478

479

480

481

482

483

484

485

486

487

488

489

490

Lopez P, Vidal F, Martin L, Lopez-Fernandez LA, Rual JF, Rosen BS, Cuzin F, and Rassoulzadegan M. 2002. Gene control in germinal differentiation: RNF6, a transcription regulatory protein in the mouse sertoli cell. Mol Cell Biol 22:3488-3496.

Loring GL, Christensen KC, Gerber SA, and Brenner C. 2008. Yeast Chfr homologs retard cell cycle at $\mathrm{G} 1$ and $\mathrm{G} 2 / \mathrm{M}$ via $\mathrm{Ubc} 4$ and Ubc13/Mms2-dependent ubiquitination. Cell Cycle 7:96-105.

Mahani A, Henriksson J, and Wright AP. 2013. Origins of Myc proteins--using intrinsic protein disorder to trace distant relatives. PLoS One 8:e75057. 10.1371/journal.pone.0075057

McEwan IJ. 2012. Intrinsic disorder in the androgen receptor: identification, characterisation and drugability. Mol Biosyst 8:82-90. 10.1039/c1mb05249g

Merlini L, Fraschini R, Boettcher B, Barral Y, Lucchini G, and Piatti S. 2012. Budding yeast dma proteins control septin dynamics and the spindle position checkpoint by promoting the recruitment of the Elm1 kinase to the bud neck. PLoS Genet 8:e1002670. 10.1371/journal.pgen.1002670

Meszaros B, Simon I, and Dosztanyi Z. 2009. Prediction of protein binding regions in disordered proteins. PLoS Comput Biol 5:e1000376. 10.1371/journal.pcbi.1000376

Metzger MB, Pruneda JN, Klevit RE, and Weissman AM. 2014. RING-type E3 ligases: master manipulators of E2 ubiquitin-conjugating enzymes and ubiquitination. Biochim Biophys Acta 1843:47-60. 10.1016/j.bbamcr.2013.05.026

Miyazaki K, Fujita T, Ozaki T, Kato C, Kurose Y, Sakamoto M, Kato S, Goto T, Itoyama Y, Aoki M, and Nakagawara A. 2004. NEDL1, a novel ubiquitin-protein isopeptide ligase for dishevelled-1, targets mutant superoxide dismutase-1. J Biol Chem 279:11327-11335. 10.1074/jbc.M312389200

Nielsen SV, Poulsen EG, Rebula CA, and Hartmann-Petersen R. 2014. Protein quality control in the nucleus. Biomolecules 4:646-661. 10.3390/biom4030646

Oshiro S, and Takagi H. 2014. The transcriptional activator Pog1 controls cell cycle, and its phosphorylated form is downregulated by the ubiquitin ligase Dma2 in Saccharomyces cerevisiae. FEMS Yeast Res 14:1015-1027. 10.1111/1567-1364.12190

Park SH, Kukushkin Y, Gupta R, Chen T, Konagai A, Hipp MS, Hayer-Hartl M, and Hartl FU. 2013. PolyQ proteins interfere with nuclear degradation of cytosolic proteins by sequestering the Sis1p chaperone. Cell 154:134-145. 10.1016/j.cell.2013.06.003

Petrovich A, Borne A, Uversky VN, and Xue B. 2015. Identifying Similar Patterns of Structural Flexibility in Proteins by Disorder Prediction and Dynamic Programming. Int J Mol Sci 16:13829-13849. 10.3390/ijms160613829

Plechanovova A, Jaffray EG, McMahon SA, Johnson KA, Navratilova I, Naismith JH, and Hay RT. 2011. Mechanism of ubiquitylation by dimeric RING ligase RNF4. Nat Struct Mol Biol 18:1052-1059. 10.1038/nsmb.2108

Potenza E, Di Domenico T, Walsh I, and Tosatto SC. 2015. MobiDB 2.0: an improved database of intrinsically disordered and mobile proteins. Nucleic Acids Res 43:D315-320. 10.1093/nar/gku982

Poulsen SL, Hansen RK, Wagner SA, van Cuijk L, van Belle GJ, Streicher W, Wikstrom M, Choudhary C, Houtsmuller AB, Marteijn JA, Bekker-Jensen S, and Mailand N. 2013. RNF111/Arkadia is a SUMO-targeted ubiquitin ligase that facilitates the DNA damage response. J Cell Biol 201:797-807. 10.1083/jcb.201212075 
491

492

493

494

495

496

497

498

499

500

501

502

503

504

505

506

507

508

509

510

511

512

513

514

515

516

517

518

519

520

521

522

523

524

525

526

527

528

529

530

531

532

533

534
Prudden J, Pebernard S, Raffa G, Slavin DA, Perry JJ, Tainer JA, McGowan CH, and Boddy MN. 2007. SUMO-targeted ubiquitin ligases in genome stability. EMBO J 26:4089-4101. $10.1038 /$ sj.emboj.7601838

Ravid T, and Hochstrasser M. 2007. Autoregulation of an E2 enzyme by ubiquitin-chain assembly on its catalytic residue. Nat Cell Biol 9:422-427. 10.1038/ncb1558

Ravid T, Kreft SG, and Hochstrasser M. 2006. Membrane and soluble substrates of the Doa10 ubiquitin ligase are degraded by distinct pathways. $E M B O \quad J$ 25:533-543. 10.1038/sj.emboj.7600946

Rodrigo-Brenni MC, Gutierrez E, and Hegde RS. 2014. Cytosolic quality control of mislocalized proteins requires RNF126 recruitment to Bag6. Mol Cell 55:227-237. 10.1016/j.molcel.2014.05.025

Rosenbaum JC, Fredrickson EK, Oeser ML, Garrett-Engele CM, Locke MN, Richardson LA, Nelson ZW, Hetrick ED, Milac TI, Gottschling DE, and Gardner RG. 2011. Disorder targets misorder in nuclear quality control degradation: a disordered ubiquitin ligase directly recognizes its misfolded substrates. Mol Cell 41:93-106. S1097-2765(10)00960-3 [pii]

10.1016/j.molcel.2010.12.004

Rosenbaum JC, and Gardner RG. 2011. How a disordered ubiquitin ligase maintains order in nuclear protein homeostasis. Nucleus 2:264-270. 16118 [pii]

Sriramachandran AM, and Dohmen RJ. 2014. SUMO-targeted ubiquitin ligases. Biochim Biophys Acta 1843:75-85. 10.1016/j.bbamcr.2013.08.022

Tammsalu T, Matic I, Jaffray EG, Ibrahim AF, Tatham MH, and Hay RT. 2014. Proteome-wide identification of SUMO2 modification sites. Sci Signal 7:rs2. 10.1126/scisignal.2005146

Tanaka H, Takahashi T, Xie Y, Minami R, Yanagi Y, Hayashishita M, Suzuki R, Yokota N, Shimada M, Mizushima T, Kuwabara N, Kato R, and Kawahara H. 2015. A conserved island of BAG6/Scythe is related to ubiquitin domains and participates in short hydrophobicity recognition. FEBS J. 10.1111/febs.13618

Tatham MH, Geoffroy MC, Shen L, Plechanovova A, Hattersley N, Jaffray EG, Palvimo JJ, and Hay RT. 2008. RNF4 is a poly-SUMO-specific E3 ubiquitin ligase required for arsenicinduced PML degradation. Nat Cell Biol 10:538-546. 10.1038/ncb1716

Tatham MH, Matic I, Mann M, and Hay RT. 2011. Comparative proteomic analysis identifies a role for SUMO in protein quality control. Sci Signal 4:rs4. 10.1126/scisignal.2001484

Theodoraki MA, Nillegoda NB, Saini J, and Caplan AJ. 2012. A network of ubiquitin ligases is important for the dynamics of misfolded protein aggregates in yeast. J Biol Chem 287:23911-23922. 10.1074/jbc.M112.341164

Tompa P. 2002. Intrinsically unstructured proteins. Trends Biochem Sci 27:527-533.

Tursun B, Schluter A, Peters MA, Viehweger B, Ostendorff HP, Soosairajah J, Drung A, Bossenz M, Johnsen SA, Schweizer M, Bernard O, and Bach I. 2005. The ubiquitin ligase Rnf6 regulates local LIM kinase 1 levels in axonal growth cones. Genes Dev 19:2307-2319. 10.1101/gad.1340605

Uzunova K, Gottsche K, Miteva M, Weisshaar SR, Glanemann C, Schnellhardt M, Niessen M, Scheel H, Hofmann K, Johnson ES, Praefcke GJ, and Dohmen RJ. 2007. Ubiquitindependent proteolytic control of SUMO conjugates. J Biol Chem 282:34167-34175. 10.1074/jbc.M706505200 
561

562

563

564

565

566
Vittal V, Shi L, Wenzel DM, Scaglione KM, Duncan ED, Basrur V, Elenitoba-Johnson KS, Baker D, Paulson HL, Brzovic PS, and Klevit RE. 2015. Intrinsic disorder drives Nterminal ubiquitination by Ube2w. Nat Chem Biol 11:83-89. 10.1038/nchembio.1700

Vogt B, and Hofmann K. 2012. Bioinformatical detection of recognition factors for ubiquitin and SUMO. Methods Mol Biol 832:249-261. 10.1007/978-1-61779-474-2_18

Wang Q, Liu Y, Soetandyo N, Baek K, Hegde R, and Ye Y. 2011. A ubiquitin ligase-associated chaperone holdase maintains polypeptides in soluble states for proteasome degradation. Mol Cell 42:758-770. 10.1016/j.molcel.2011.05.010

Wang X, Herr RA, and Hansen TH. 2012. Ubiquitination of substrates by esterification. Traffic 13:19-24. 10.1111/j.1600-0854.2011.01269.x

Wang Z, Jones GM, and Prelich G. 2006. Genetic analysis connects SLX5 and SLX8 to the SUMO pathway in Saccharomyces cerevisiae. Genetics 172:1499-1509. 10.1534/genetics.105.052811

Ward JJ, Sodhi JS, McGuffin LJ, Buxton BF, and Jones DT. 2004. Prediction and functional analysis of native disorder in proteins from the three kingdoms of life. $\mathrm{J} \mathrm{Mol} \mathrm{Biol}$ 337:635-645. 10.1016/j.jmb.2004.02.002

Westerbeck JW, Pasupala N, Guillotte M, Szymanski E, Matson BC, Esteban C, and Kerscher O. 2014. A SUMO-targeted ubiquitin ligase is involved in the degradation of the nuclear pool of the SUMO E3 ligase Siz1. Mol Biol Cell 25:1-16. 10.1091/mbc.E13-05-0291

Xie Y, and Varshavsky A. 2002. UFD4 lacking the proteasome-binding region catalyses ubiquitination but is impaired in proteolysis. Nat Cell Biol 4:1003-1007. 10.1038/ncb889

Xu K, Shimelis H, Linn DE, Jiang R, Yang X, Sun F, Guo Z, Chen H, Li W, Chen H, Kong X, Melamed J, Fang S, Xiao Z, Veenstra TD, and Qiu Y. 2009. Regulation of androgen receptor transcriptional activity and specificity by RNF6-induced ubiquitination. Cancer Cell 15:270-282. 10.1016/j.ccr.2009.02.021

Xu Y, Liu Y, Lee JG, and Ye Y. 2013. A ubiquitin-like domain recruits an oligomeric chaperone to a retrotranslocation complex in endoplasmic reticulum-associated degradation. $J$ Biol Chem 288:18068-18076. 10.1074/jbc.M112.449199

Yang L, Mullen JR, and Brill SJ. 2006. Purification of the yeast Slx5-Slx8 protein complex and characterization of its DNA-binding activity. Nucleic Acids Res 34:5541-5551. $10.1093 / \mathrm{nar} / \mathrm{gk} 1685$ 


\section{Figure 1 (on next page)}

San1 is highly disordered and contains a characteristic pattern of interspersed ANCHOR positive regions for substrate interaction.

(A) Disorder annotation of the S. cerevisiae San1 protein, as provided by the MobiDB database (Potenza et al. 2015). Nine individual predictors were used by MobiDB as the basis for a consensus annotation, which we use for all analyses in this paper. The darkness of the shade of blue indicates how many of the nine predictors agree about the disorder annotation at a particular site. (B) Combined plot of the MobiDB consensus disorder annotation (blue) and the ANCHOR prediction (red) for San1. The position of the RING domain is indicated. This compact view is used as the basis for the comparative analyses throughout the paper. 
DisEMBL-465

DisEMBL-HL

ESpritz-DisProt

ESpritz-NMR

ESpritz-XRay

IUPred-long

IUPred-short

JRONN

VSL2b

B

Consensus + Anchor

Manuscript to be reviewed

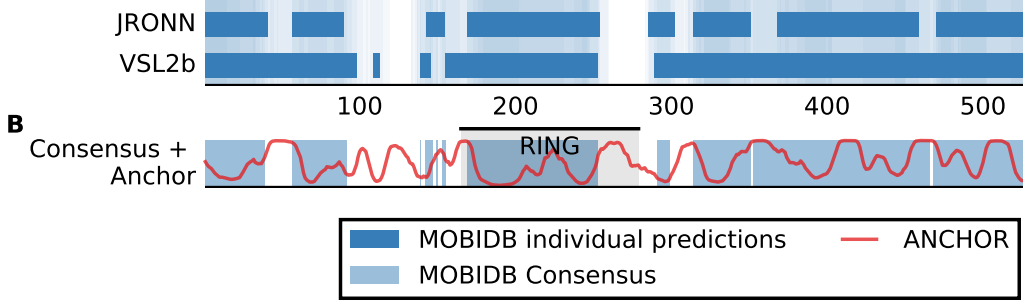


Figure 2 (on next page)

Characterization of the E3 dataset in terms of disorder and protein binding sites in disordered regions.

Plots of ANCHOR score versus fraction consensus disorder for all S. cerevisiae (A) and human

(B) proteins in the datasets. Protein names are given for the top-scoring candidates, for which disorder and ANCHOR profiles are depicted in Figure 3. 
A

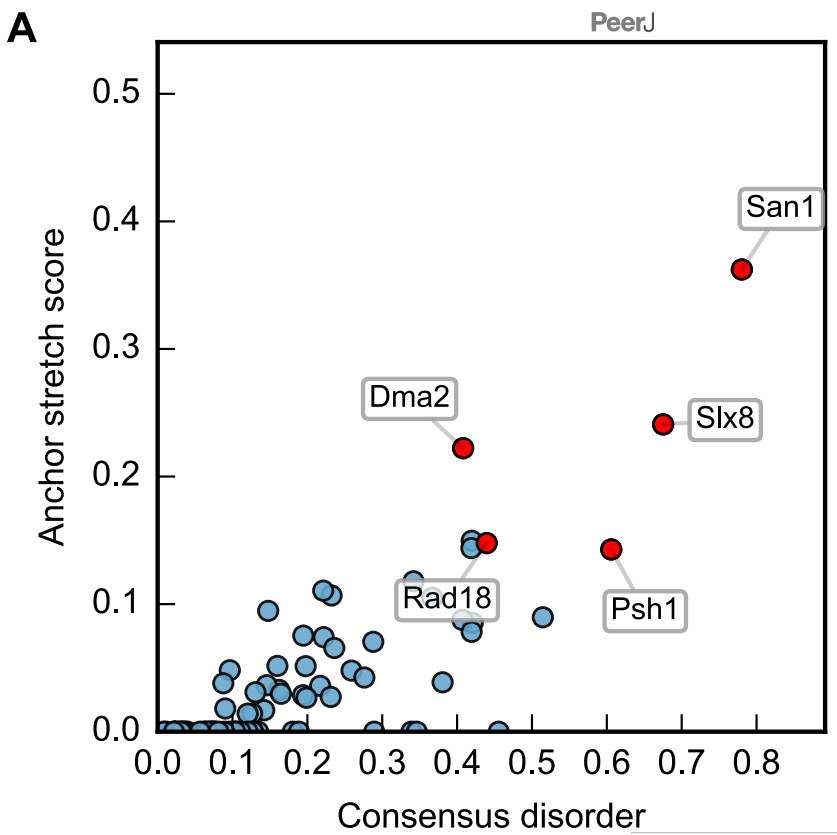

B

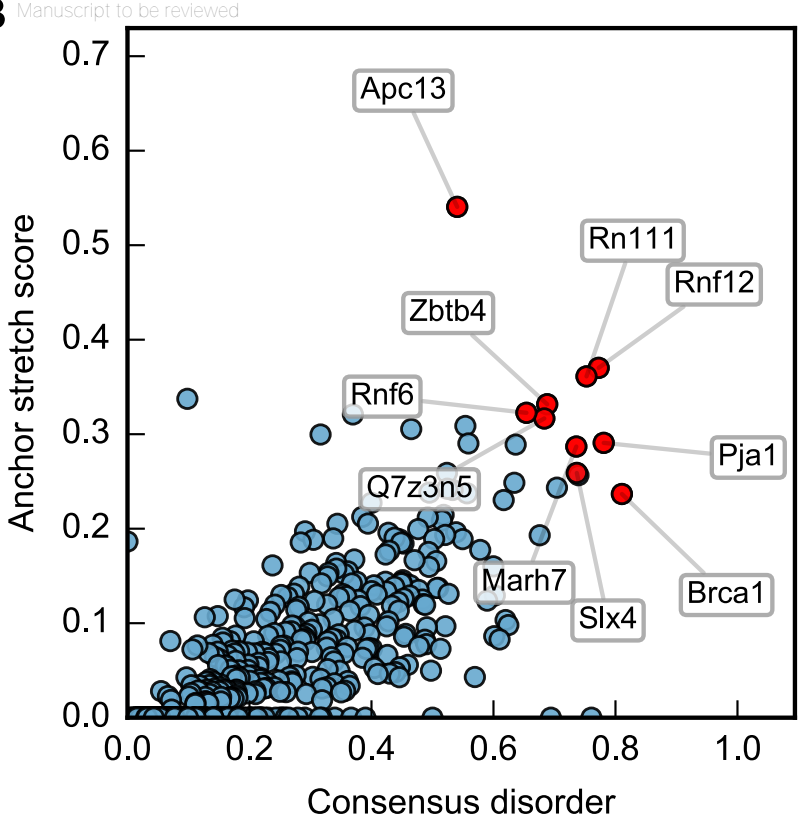




\section{Figure 3 (on next page)}

Identified San1-like E3 ligases show unusually low lysine content outside of the RING domain.

Plots of the highest-scoring candidates obtained using a score consisting of the product of disorder and anchor-stretch score. Each occurrence of a lysine residue is marked with a black dot. 


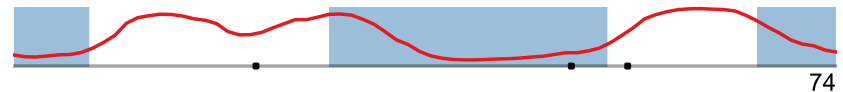

3. Rn111 (Q6ZNA4)

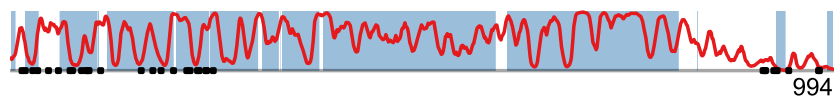

5. Pja1 (Q8NG27)

hhman

7. Marh7 (Q9H992)

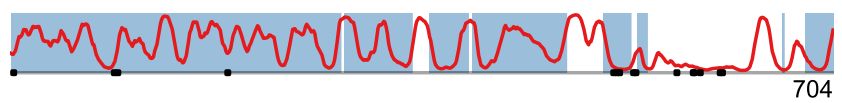

9. Brca1 (P38398)

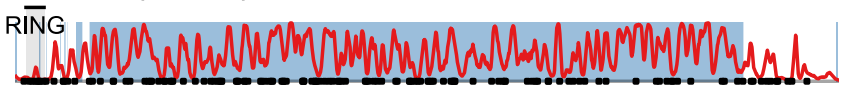

1. San1 (P22470)

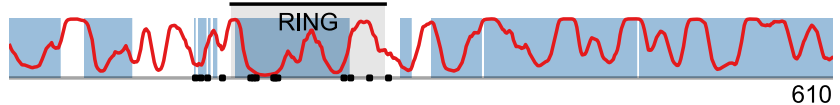

3. Dma2 (P53924)

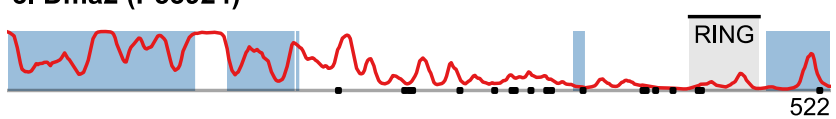

5. Rad18 (P10862)

$\overline{\mathrm{RING}}$

mhly

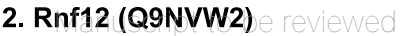

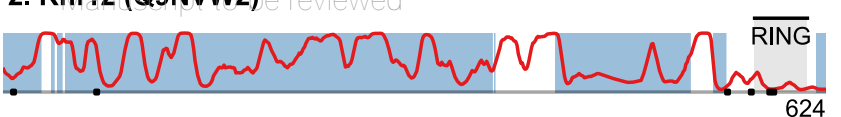

\section{Zbtb4 (Q9P1Z0)}

M ${ }^{\mathrm{BTB}} \mathrm{M}$

\section{Q7z3n5 (Q7Z3N5)}

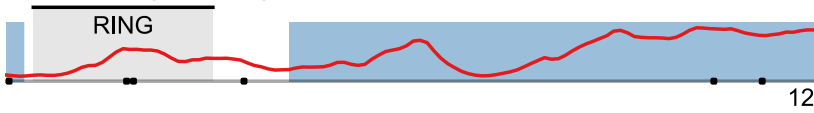

\section{Rnf6 (Q9Y252)}

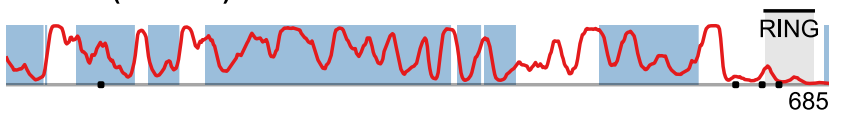

10. SIX4 (Q8IY92)

.NW WMM MNMW WW

2. SIx8 (P40072)

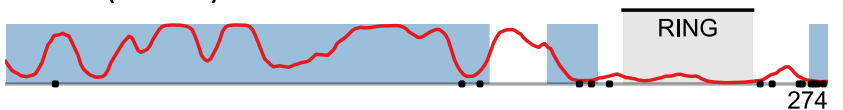

4. Psh1 (Q12161)

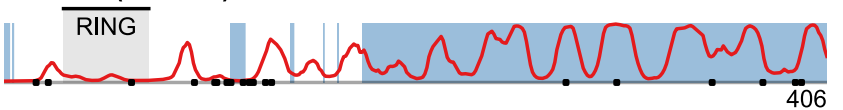




\section{Figure 4 (on next page)}

Low lysine content in San1 candidates is conserved broadly across species.

The lysine distributions of the candidates with strongest lysine suppression in Figure 3 are compared to select orthologs from representative species of evolutionary divergence, suggesting lysine suppression to be a strongly conserved feature in these candidates. 


\section{Rnf12 (Q9NVW2)}

Human

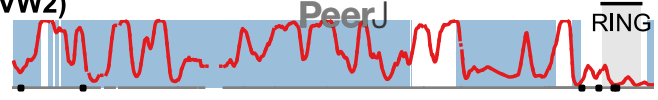

Sheep -

Cow .

Elephant -

Hyrax -

Megabat .

Bushbaby -.

Turkey .

Zebrafish

\section{Rnf6 (Q9Y252)}

Human

Bushbaby

Megabat

Elephant

Cow

Sheep

Hyrax

Turkey

Zebrafish

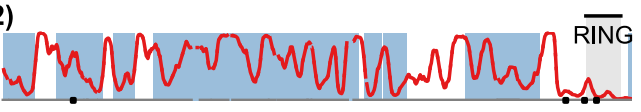

\section{SIx8 (P40072)}

S. cerevisiae

S. kudriavzevii

S. arboricola

S. arboricola

S. octosporus

S. cryophilus S. pombe

S. japonicus

$U$. necator
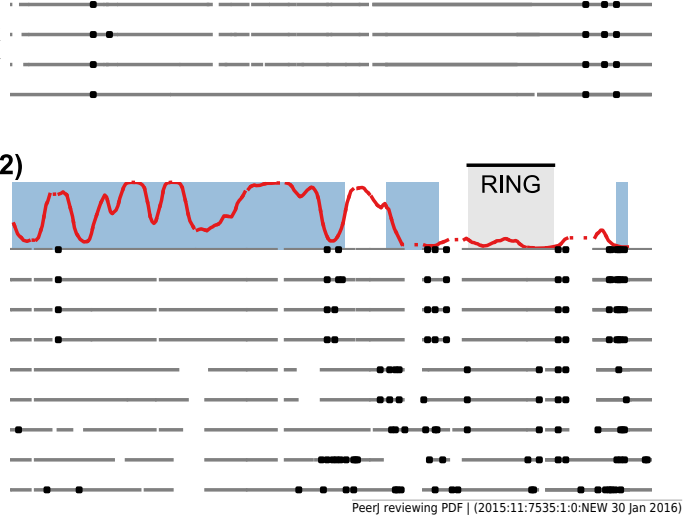

Human

Bushbaby

Megabat

Cow

Sheep

Elephant

Turkey

Hyrax

\section{Marh7 (Q9H992)}

Human

Bushbaby --

Megabat - - -

Elephant - Cow --

Sheep -

Hyrax - -

Turkey - - -

Zebrafish $\bullet$.
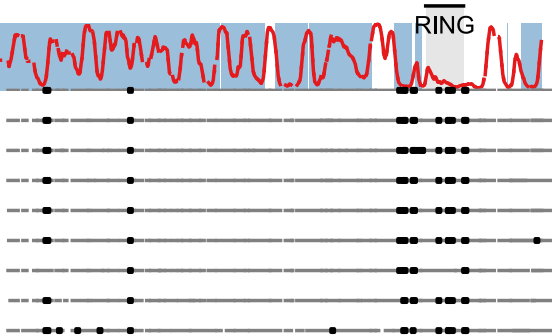

$\begin{array}{lll}\text { MOBIDB Consensus } & \cdots & \text { Lysine residues } \\ & \text { ANCHOR }\end{array}$ 


\section{Figure $\mathbf{5}$ (on next page)}

San1-like candidates can be detected using a feature-based similarity score.

Top-scoring candidates retrieved when applying the sliding-window San1-similarity score (see Table S1). Three complementary quantities are calculated over a window of 150 residues: 1) the percentage of disorder, 2) the percentage of residues within an ANCHOR positive stretch of length 15-40 and 3) the percentage of residues occurring in the maximum lysine-free stretch within the window. The San1-similarity score is the maximum value of the product of these numbers. The window size is chosen to be of fixed size, and the score is therefore not defined for the first and last 75 positions. Note that certain of the top-scoring candidates found using the San1 similarity score overlap with our initial, simpler approach that considered only disorder and ANCHOR score over the entire sequence (see Figure 3). 
